\title{
Correction to: Synthesis and antibacterial activity of novel chalcone derivatives bearing a coumarin moiety
}

\author{
Yi-Hui Wang $^{1} \cdot$ Shi-Chun Jiang ${ }^{1} \cdot$ Ying Chen $^{1} \cdot$ Tao Guo $^{1} \cdot$ Rong-Jiao Xia $^{1} \cdot$ Xu Tang $^{1} \cdot$ Ming He $^{1} \cdot$ Wei Xue $^{1}$
}

Published online: 23 July 2020

(c) Institute of Chemistry, Slovak Academy of Sciences 2020

\section{Correction to: Chemical Papers (2019) 73:2493-2500 https://doi.org/10.1007/s11696-019-00802-0}

Unfortunately the original article was published Online with incorrect entries as given below in the Table 3 .

Table 3 was submitted for publication with numerous incorrect entries. \%Inhibition values for the entries for compound $\mathbf{3 l}$ at $50 \mu \mathrm{g} \mathrm{mL} \mathrm{m}^{-1}$ against Xoo, and for 4-hydroxycoumarin and for compounds $\mathbf{3 a}-\mathbf{3 v}$ against Xac, are corrected. The values for thiodiazole-copper control in Table 3 essentially are unchanged. Where the difference between the corrected value and the previous incorrect value is greater than two-fold, both the correct and the previously reported incorrect values are listed here. The revised Table 3 given below shows all entries, each with their correct values. The corrected values are for $\mathbf{3 l}$, \% Inhibition at $50 \mu \mathrm{g} \mathrm{mL}^{-1}$ against Xoo is $19 \pm 3$; for 4-hydroxycoumarin, \% Inhibition at $50 \mu \mathrm{g} \mathrm{mL}^{-1}$ against Xac is $21 \pm 4$ and \% Inhibition at $100 \mu \mathrm{g} \mathrm{mL} L^{-1}$ against $\mathrm{Xac}$ is $29 \pm 4$. The corrected values for \% Inhibition at $50 \mu \mathrm{g} \mathrm{mL} \mathrm{m}^{-1}$ against Xac are: $\mathbf{3 a}(4 \pm 4$, previously was $47 \pm 2)$; $3 \mathbf{b}(18 \pm 1)$; $3 \mathbf{c}(34 \pm 3)$; $\mathbf{3 d}(19 \pm 4)$; 3e $(41 \pm 2$, previously was $4 \pm 2) ; \mathbf{3 f}(13 \pm 5$, previously was $6 \pm 5) ; \mathbf{3 g}(9 \pm 4$, previously was $4 \pm 1) ; \mathbf{3 h}(3 \pm 5$, previously was $1 \pm 4) ; \mathbf{3 i}(34 \pm 3) ; \mathbf{3 j}(19 \pm 3$, previously was $51 \pm 4)$; 3k (33 \pm 3$)$; $3 \mathbf{l}$ (22 \pm 5$)$; 3m (29 \pm 4 , previously was $6 \pm 5)$; 3n $(54 \pm 4$, previously was $10 \pm 2)$; 3 o $(37 \pm 1$, previously was $14 \pm 1)$; $3 \mathbf{p}(45 \pm 4$, previously was $5 \pm 2)$; $3 \mathbf{q}(9 \pm 3$,

The original article can be found online at https://doi.org/10.1007/ s11696-019-00802-0.

Ming He

mhe1@gzu.edu.cn

Wei Xue

wxue@gzu.edu.cn

1 State Key Laboratory Breeding Base of Green Pesticide and Agricultural Bioengineering, Key Laboratory of Green Pesticide and Agricultural Bioengineering, Ministry of Education, Guizhou University, Huaxi District, Guiyang 550025, China previously was $32 \pm 7)$; $3 \mathbf{r}(19 \pm 3)$; $3 \mathbf{s}(36 \pm 3$, previously was $6 \pm 2) ; 3 \mathbf{t}$ (33 \pm 4 , previously was $12 \pm 4) ; \mathbf{3 u}(7 \pm 1$, previously was $20 \pm 3) ; \mathbf{3 v}(27 \pm 1$, previously was $5 \pm 1)$. The corrected values are \% Inhibition at $100 \mu \mathrm{g} \mathrm{mL}^{-1}$ against Xac: 3a (10 \pm 3 , previously was $71 \pm 2)$; $3 \mathbf{b}(22 \pm 2$, previously was $46 \pm 4)$; $3 \mathbf{c}(49 \pm 4)$; $3 \mathbf{d}(33 \pm 5)$; $3 \mathbf{e}(72 \pm 3$, previously was $6 \pm 3)$; $\mathbf{3 f}(30 \pm 1)$; $\mathbf{3 g}(23 \pm 1)$; $\mathbf{3 h}(9 \pm 4) ; \mathbf{3 i}$ $(58 \pm 2$, previously was $23 \pm 2) ; \mathbf{3 j}(34 \pm 2) ; \mathbf{3 k}(57 \pm 5)$; $\mathbf{3 l}$ (28 \pm 4$)$; 3m (54 \pm 3 , previously was $10 \pm 5)$; 3n (72 \pm 4 , previously was $13 \pm 3)$; $30(60 \pm 3$, previously was $17 \pm 4)$; 3p $(78 \pm 5$, previously was $7 \pm 4)$; 3q $(18 \pm 3$, previously was $48 \pm 2)$; $3 \mathbf{r}(29 \pm 3)$; $3 \mathbf{s}(50 \pm 4$, previously was $9 \pm 2)$; 3t $(60 \pm 1$, previously was $17 \pm 3)$; $3 \mathbf{u}(16 \pm 2)$; $\mathbf{3 v}(47 \pm 4$, previously was $12 \pm 2$ ).

The revision of these data in Table 3 does not affect the conclusions of this paper. Its conclusions were based on the $\mathrm{EC}_{50}$ data of Table 4. The $\mathrm{EC}_{50}$ data were obtained by a separate experiment from the experiment used for the \% Inhibition data of Table 3 (as stated on p. 2497). From the data in Table 4 on p. 2499, and the statements in the fourth paragraph on pp. 2497 and 2498 ("Compounds 3c, 3e, 3i, 3k, 3m, 3n, 3o, 3p, 3s, 3t and 3v expressed strong antibacterial activity against $\mathrm{Xac}$, with $\mathrm{EC}_{50}$ values of $98,59,80$, $83,94,48,72,51,94,77$, and $119 \mu \mathrm{g} / \mathrm{mL}$, respectively"), compounds $\mathbf{3 e}, \mathbf{3 n}$, and $\mathbf{3 p}$ are identified as the most active compounds against Xac. The corrected data of Table 3 better support this conclusion. Moreover, the corrected data also support the statement (third paragraph on page 2497) that "Compounds 3c, 3e, 3i, 3k, 3m, 3n, 3o, 3p, 3s, 3t and 3v showed qualified antibacterial activity against Xac at $100 \mu \mathrm{g} /$ $\mathrm{mL}$, and the achieved inhibition ranged from 47 to $78 \%$ ". 
Table 3 Antibacterial activities of compounds 4-hydroxycoumarin, 3a-3v, and Thiodiazole-copper

\begin{tabular}{|c|c|c|c|c|c|c|}
\hline \multirow[t]{3}{*}{ Compound } & \multicolumn{6}{|c|}{ Inhibition rate $(\%)^{\mathrm{a}}$} \\
\hline & \multicolumn{2}{|l|}{ Хоо } & \multicolumn{2}{|l|}{$X a c$} & \multicolumn{2}{|l|}{ Rs } \\
\hline & $100 \mu \mathrm{g} / \mathrm{mL}$ & $50 \mu \mathrm{g} / \mathrm{mL}$ & $100 \mu \mathrm{g} / \mathrm{mL}$ & $50 \mu \mathrm{g} / \mathrm{mL}$ & $100 \mu \mathrm{g} / \mathrm{mL}$ & $50 \mu \mathrm{g} / \mathrm{mL}$ \\
\hline 4-Hydroxy-coumarin & $23 \pm 4$ & $15 \pm 4$ & $29 \pm 4$ & $21 \pm 4$ & $16 \pm 3$ & $9 \pm 2$ \\
\hline $3 \mathbf{a}$ & $71 \pm 2$ & $47 \pm 2$ & $10 \pm 3$ & $4 \pm 4$ & $39 \pm 1$ & $20 \pm 4$ \\
\hline $3 \mathbf{b}$ & $46 \pm 4$ & $29 \pm 3$ & $22 \pm 2$ & $18 \pm 1$ & $12 \pm 2$ & $7 \pm 3$ \\
\hline $3 \mathbf{c}$ & $42 \pm 5$ & $34 \pm 5$ & $49 \pm 4$ & $34 \pm 3$ & $18 \pm 2$ & $16 \pm 1$ \\
\hline 3d & $46 \pm 3$ & $26 \pm 5$ & $33 \pm 5$ & $19 \pm 4$ & $20 \pm 2$ & $8 \pm 1$ \\
\hline $3 \mathbf{e}$ & $6 \pm 3$ & $4 \pm 2$ & $72 \pm 3$ & $41 \pm 2$ & $28 \pm 5$ & $24 \pm 3$ \\
\hline $3 f$ & $21 \pm 2$ & $6 \pm 5$ & $30 \pm 1$ & $13 \pm 5$ & $29 \pm 3$ & $26 \pm 2$ \\
\hline $3 \mathbf{g}$ & $18 \pm 5$ & $4 \pm 1$ & $23 \pm 1$ & $9 \pm 4$ & $14 \pm 3$ & $8 \pm 4$ \\
\hline $3 \mathbf{h}$ & $6 \pm 2$ & $1 \pm 4$ & $9 \pm 4$ & $3 \pm 5$ & $20 \pm 3$ & $11 \pm 5$ \\
\hline $3 \mathbf{i}$ & $23 \pm 2$ & $17 \pm 2$ & $58 \pm 2$ & $34 \pm 3$ & $66 \pm 5$ & $49 \pm 3$ \\
\hline $3 \mathbf{j}$ & $60 \pm 3$ & $51 \pm 4$ & $34 \pm 2$ & $19 \pm 3$ & $32 \pm 3$ & $27 \pm 5$ \\
\hline $3 \mathbf{k}$ & $39 \pm 5$ & $26 \pm 4$ & $57 \pm 5$ & $33 \pm 3$ & $48 \pm 6$ & $33 \pm 2$ \\
\hline $3 \mathbf{l}$ & $40 \pm 4$ & $19 \pm 3$ & $28 \pm 4$ & $22 \pm 5$ & $15 \pm 7$ & $7 \pm 1$ \\
\hline $3 \mathrm{~m}$ & $10 \pm 5$ & $6 \pm 5$ & $54 \pm 3$ & $29 \pm 4$ & $23 \pm 2$ & $22 \pm 3$ \\
\hline $3 n$ & $13 \pm 3$ & $10 \pm 2$ & $72 \pm 4$ & $54 \pm 4$ & $11 \pm 2$ & $1 \pm 5$ \\
\hline 30 & $17 \pm 4$ & $14 \pm 1$ & $60 \pm 3$ & $37 \pm 1$ & $13 \pm 3$ & $0 \pm 4$ \\
\hline $3 \mathbf{p}$ & $7 \pm 4$ & $5 \pm 2$ & $78 \pm 5$ & $45 \pm 4$ & $38 \pm 5$ & $12 \pm 1$ \\
\hline $3 \mathbf{q}$ & $48 \pm 2$ & $32 \pm 7$ & $18 \pm 3$ & $9 \pm 3$ & $15 \pm 2$ & $1 \pm 3$ \\
\hline $3 \mathbf{r}$ & $24 \pm 1$ & $22 \pm 1$ & $29 \pm 3$ & $19 \pm 3$ & $20 \pm 4$ & $15 \pm 6$ \\
\hline $3 \mathbf{s}$ & $9 \pm 2$ & $6 \pm 2$ & $50 \pm 4$ & $36 \pm 3$ & $36 \pm 6$ & $28 \pm 1$ \\
\hline $3 t$ & $17 \pm 3$ & $12 \pm 4$ & $60 \pm 1$ & $33 \pm 4$ & $26 \pm 1$ & $23 \pm 6$ \\
\hline $3 \mathbf{u}$ & $29 \pm 5$ & $20 \pm 3$ & $16 \pm 2$ & $7 \pm 1$ & $32 \pm 3$ & $30 \pm 5$ \\
\hline $3 \mathbf{v}$ & $12 \pm 2$ & $5 \pm 1$ & $47 \pm 4$ & $27 \pm 1$ & $12 \pm 2$ & $1 \pm 5$ \\
\hline Thiodiazole-copper $^{\mathrm{b}}$ & $36 \pm 3$ & $26 \pm 4$ & $37 \pm 4$ & $26 \pm 4$ & $41 \pm 4$ & $24 \pm 2$ \\
\hline
\end{tabular}

${ }^{a}$ Average of three replicates

${ }^{\mathrm{b}}$ The commercial antibacterial agent Thiodiazole-copper was used as positive control

Publisher's Note Springer Nature remains neutral with regard to jurisdictional claims in published maps and institutional affiliations. 